\title{
Diversity in NAFLD: A Review of Manifestations of Nonalcoholic Fatty Liver Disease in Different Ethnicities Globally
}

\author{
Ma Ai Thanda Han®, Qi Yu, Zaid Tafesh and Nikolaos Pyrsopoulos*(1) \\ Division of Gastroenterology and Hepatology, Rutgers New Jersey Medical School, Newark, NJ 07103, USA
}

\begin{abstract}
Globally, the rise in prevalence of obesity and metabolic syndrome as a whole has been linked to increased access to processed foods, such as refined sugars and saturated fats. Consequently, nonalcoholic fatty liver disease (NAFLD) is on the rise in both developed and developing nations. However, much is still unknown on the NAFLD phenotype with regards to the effect of ethnic diversity. Despite similarities in dietary habits, it appears that certain ethnicities are more protected against NAFLD than others. However, manifestations of the same genetic polymorphisms in different groups of people increase those individuals' predisposition to NAFLD. Diets from different regions have been associated with a lower prevalence of NAFLD and have even been linked to regression of hepatic steatosis. Socioeconomic variations amongst different regions of the world also contribute to NAFLD prevalence and associated complications. Thus, a thorough understanding of ethnic variability in NAFLD is essential to tailoring treatment recommendations to patients of different backgrounds.
\end{abstract}

Citation of this article: Han MAT, Yu Q, Tafesh Z, Pyrsopoulos N. Diversity in NAFLD: A review of manifestations of nonalcoholic fatty liver disease in different ethnicities globally. J Clin Transl Hepatol 2021;000(000):000-000. doi: 10.14218/JCTH.2020.00082.

\section{Introduction}

The current landscape of liver disease has evolved over the last few decades. With the advent of highly effective therapy for viral hepatitis, nonalcoholic fatty liver disease (NAFLD) has become one of the most common etiologies of chronic liver disease. ${ }^{1}$ NAFLD is defined by detection of hepatic steatosis,

Keywords: Nonalcoholic fatty liver; Nonalcoholic steatohepatitis; Ethnicity; Diversity; Body mass index; Lean.

Abbreviations: APRI, aspartate aminotransferase/platelet ratio index; ARFI acoustic radiation force impulses; BMI, body mass index; CAP, controlled attenuation parameter; DM, diabetes mellitus; FIB-4, fibrosis-4 index; FLI, fatty liver index; GWAS, genome-wide association studies; HCC, hepatocellular carcinoma; HIS, hepatitis steatosis index; MAFLD, metabolic dysfunction-associated fatty liver disease; MRE, magnetic resonance elastography; MS, metabolic syndrome; NAFLD, nonalcoholic fatty liver; NASH, nonalcoholic steatohepatitis; NHANES, National Health and Nutrition Examination Survey; PNPLA3, patatinlike phospholipase domain-containing protein 3; $\mathrm{TE}$, transient elastography. Received: 25 August 2020; Revised: 17 November 2020; Accepted: 05 December 2020

*Correspondence to: Nikolaos Pyrsopoulos, Division of Gastroenterology and Hepatology, Rutgers New Jersey Medical School, 185 South Orange Avenue, H-536, Newark, NJ 07103, USA. Tel: +1-973-972-5252, E-mail: pyrsopni@njms. rutgers.edu or the presence of macrovesicular fat in $>5 \%$ of hepatocytes, either by imaging or histology, after exclusion of secondary causes and alcoholic fatty liver disease. ${ }^{2}$ Currently, international experts consensus have proposed the more comprehensive and practical nomenclature of "metabolic dysfunction-associated fatty liver disease (MAFLD)", which is defined by evidence of hepatic steatosis plus one of the following three criteria: overweight/obesity, presence of type 2 diabetes mellitus (DM), or evidence of metabolic dysregulation. 3,4

The increased prevalence of NAFLD is driven in large part by the increasing presence of metabolic syndrome, obesity, sedentary lifestyle, and improved access to food supplies globally. ${ }^{5}$ The effect is seen not only in adults but also in children and adolescents. ${ }^{6}$ This review will focus primarily on ethnic differences in NAFLD prevalence and its socioeconomic effect, in addition to risk factors, manifestations, diagnosis, and outcomes.

\section{Diagnosis and manifestations}

NAFLD is a spectrum of disease continuity, ranging from simple steatosis, steatohepatitis (NASH) with or without different stages of fibrosis, cirrhosis, and hepatocellular carcinoma (HCC). ${ }^{7}$ Even though most cases of HCC develop in cirrhotic patients, there is increasing evidence of development of HCC in non-cirrhotic NASH. ${ }^{8}$ The majority of individuals with NAFLD are asymptomatic, and the diagnosis is often discovered incidentally during workup for elevated liver function tests or via imaging for another purpose. Compared to the non-NAFLD population, a higher rate of fatigue impairing physical function has been found in NAFLD/NASH patients. Energy level is found to be lower in patients with significant hepatic fibrosis compared to a normal/mild hepatic fibrosis group. ${ }^{9,10}$ Pruritus has also been reported among NASH patients. ${ }^{11}$ Other symptoms include abdominal bloating/swelling, abdominal discomfort, sleep disturbance, or apnea. ${ }^{12}$ There are no data on difference in presentations amongst various ethnicities.

NAFLD is most often diagnosed non-invasively with abdominal imaging; although, a liver biopsy is the gold standard to determine the presence steatohepatitis and stage of fibrosis. It is worth noting that ultrasound sensitivity to NAFLD is poor when steatosis occupies less than $30 \%$ of the liver, making it an inferior modality to diagnose the condition at the $5 \%$ liver fat reference. ${ }^{13}$ However, specific biomarker panels such as the fatty liver index (FLI) as validated in Italy (AUROC 0.84$)^{14}$ and the hepatitis steatosis index (HIS) as validated in Korea (AUROC 0.81$)^{15}$ can augment ultrasonography and assist in better defining the risk of steatosis in patients of different ethnicities.

Due to the higher cost and/or health risks associated with other modalities, such as computed tomography or liver biopsy, ultrasound is primarily used to diagnose patients in 
Han M.A.T. et al: NAFLD in different ethnicities

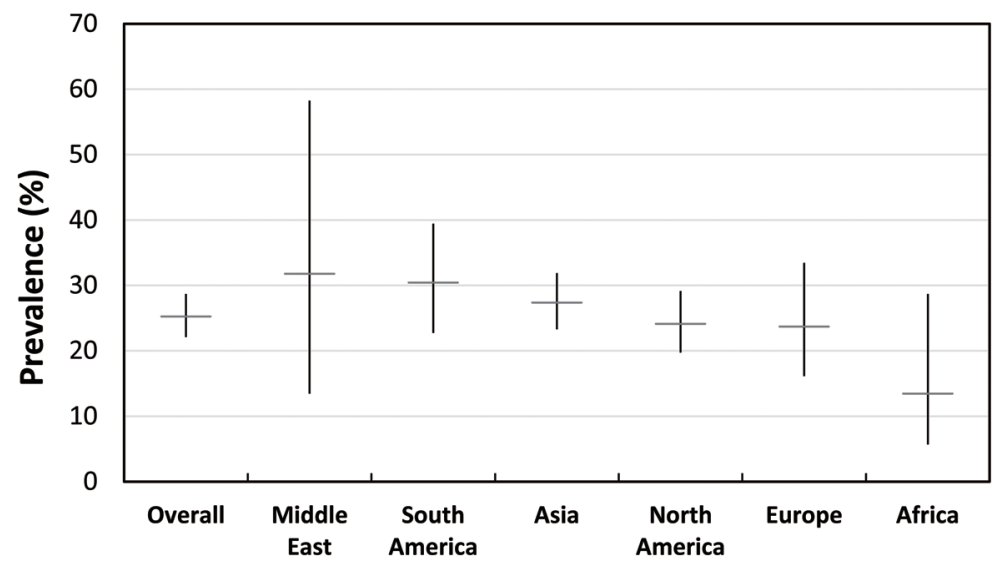

Fig. 1. Prevalence of NAFLD by geographical regions.

developing countries. ${ }^{16}$ Controlled attenuation parameter (CAP) is an ancillary tool to conventional ultrasonography and measures how hepatic fat attenuates ultrasound waves while liver stiffness is measured by transient elastography. It can assist in detecting and grading hepatic fat, although it has not been shown to reliably estimate the quantity of liver fat. ${ }^{17}$ Magnetic resonance imaging, i.e. spectroscopy or proton density fat fraction, is increasingly being used in NAFLD clinical trials to detect hepatic steatosis in both Western and Eastern countries. ${ }^{18}$ Factors that prevent magnetic resonance imaging from consideration as a first-line diagnostic tool include accessibility due to specialized equipment parts and expertise of the operator and radiologist. ${ }^{19} \mathrm{~A}$ noninvasive algorithm developed in Finland, known as the NAFLD liver fat score, used ${ }^{1} \mathrm{H}-\mathrm{MRS}$ as a reference and had an AUROC of $0.86-0.87$, but used serum insulin level as a part of its panel, which limited its accessibility to a wider patient population. ${ }^{20}$

Assessing the stage of hepatic fibrosis is important in NAFLD as it can predict the risk of disease progression and mortality. ${ }^{21}$ Non-invasive tests including serum biomarkers and imaging tests have been developed to detect hepatic fibrosis in risk stratification. Non-invasive serum biomarkers include simple tests, such as aspartate aminotransferase/ alanine aminotransferase ratio, NAFLD fibrosis score, fibrosis-4 (commonly known as FIB-4) index, and aspartate aminotransferase/platelet ratio index (commonly known as APRI), and complex tests, such as FibroTest/FibroSure, Fibrospect, enhanced liver fibrosis panel, Pro-C3 based predictive fibrosis score and NIS4, which are not commercially available yet. ${ }^{2}$ However, the simple non-invasive biomarkers are less sensitive in identifying advanced fibrosis in South Asians compared to Caucasians. ${ }^{23}$ The imaging tests including transient elastography, acoustic radiation force impulses (commonly known as ARFI), 2D shear wave elastography and magnetic resonance elastography (commonly known as MRE) have been shown to accurately detect advanced fibrosis. ${ }^{24}$ ARFI and 2D shear wave elastography imaging can reliably detect cirrhosis, although the data for transient elastography and MRE are more robust. 25,26 However, studies investigating these different modalities that were performed in patients from Asia, Europe and the USA have shown no obvious differences in performance amongst different patient ethnicities.

\section{Epidemiology}

\section{Prevalence}

The global prevalence of NAFLD varies across different re- gions. It has been estimated to be around $25.24 \%$ of the population based on data from 2016. It is highest in the Middle East followed by South America and Asia. The prevalence of NAFLD in North America and Europe is estimated around 24\%, while it was lowest in Africa (Fig. 1). ${ }^{27}$ NAFLD is known to occur between the $4^{\text {th }}$ and $6^{\text {th }}$ decades of life, ${ }^{2}$ although older age is related to higher rates of developing the disease. Traditionally, it was thought that NAFLD occurred more in men, although some studies dispute this sex bias. The median age of women who develop NAFLD is also higher than that of men. ${ }^{28}$ While studies from Thailand and Sri Lanka have found increased prevalence of NAFLD in females, in the USA, China, and Spain the prevalence was higher in men. ${ }^{29}$ Many factors, including diagnostic modalities used and inter-country variation in obesity prevalence by sex, could contribute to the sex discrepancies found among studies.

The Dallas Heart Study is a key study that explored NAFLD distribution amongst different ethnicities in the USA. ${ }^{30}$ Using magnetic resonance spectroscopy, the investigators observed that there was a significant difference amongst patients of Hispanic, Caucasian, and African American ethnicity with respect to the prevalence of NAFLD, with those of Hispanic origin being more predisposed. Similar data was also derived from the Multiethnic Cohort Study in Hawaii, although the authors reported a significantly increased prevalence in Japanese Americans. ${ }^{31}$ Individuals of Alaskan-Native ethnicity have been reported to have a NAFLD prevalence under $5 \%$, although this should be confirmed in future studies. ${ }^{32}$

Studies from both the USA and South America have established that not all patients of Hispanic origin have the same predisposition to NAFLD. While the prevalence among the overall Hispanic population has been reported as high as $29 \%$, within the USA, individuals of Hispanic ethnicity from Mexico had a significantly higher prevalence of NAFLD than patients from Puerto Rico or the Dominican Republic. ${ }^{33}$ Moreover, patients from Central America and South America also had higher a prevalence of NAFLD that was not explained by differences in rates of metabolic syndrome, physical activity, or diet. ${ }^{34}$ For example, studies from Brazil estimate the prevalence of NAFLD to be around $30 \% .{ }^{35}$ In a study of patients who underwent bariatric surgery, advanced stage of disease was likely to occur more in Hispanic $(43 \%)$ and non-Hispanic white $(46 \%)$ patients compared to non-Hispanic Black $(21 \%)$ patients. 36

In Europe, the prevalence of NAFLD varies greatly among the different countries; although similar to patients around the globe, those with metabolic syndrome had a NAFLD prevalence of around $50 \% .{ }^{37}$ It is worth noting that Eastern 
European countries such as Romania reported lower prevalence at around $20 \%$ in the same group of individuals. ${ }^{38}$ Within European countries, prevalence also has proven to vary among different ethnicities. In Greece, prevalence of NAFLD is reported to be about $41 \%$ and in Spain was found to be $33 \%$ in males and $20 \%$ in woman. ${ }^{29}$ In the UK, the highest incidence of abnormal liver tests were among patients of Bangladeshi, Pakistani, and Indian heritage. ${ }^{39}$

Interestingly, although patients of Asian ethnicity have a lower body mass index (BMI) on average than other racial groups, they continue to have a higher prevalence of NAFLD, reported as high as $29.62 \%$, which has increased significantly over the past two decades. The reported prevalence rates from Korea and Taiwan are $24-40 \%$ and 15$27 \%$ respectively, compared to Japan at $9-18 \%$. Indonesia has the highest prevalence $(51.04 \%) .^{5}$

Although literature from Africa is limited, the reported prevalence was among the lowest worldwide, with Nigeria reporting $8.7 \%$ prevalence. ${ }^{40}$ In fact, the Dallas Heart Study noted African Americans to have the lowest prevalence of NAFLD in the USA; although, they do have a higher chance of in-hospital mortality, longer hospital stay, and poorer discharge destination compared to non-Hispanic Caucasians. ${ }^{41}$ Moreover, a South African study found that progression of fibrosis in patients with NAFLD seems to occur less frequently in those of Black African ethnicity. ${ }^{42}$

A meta-analysis of the literature published on NAFLD from around the world noted that the highest prevalence of NAFLD is in South America and the Middle East. ${ }^{43}$ In the Middle East, it is estimated to be $20-30 \%$; although, wide variation appears to exist between various countries. For example, Iran reports the prevalence to be $4.1 \%$, while Saudi Arabia cites $16.6 \% .{ }^{29}$ Unfortunately, the majority of Middle Eastern countries have not published extensive studies on NAFLD and inconsistencies in regulation, practice, and reporting have affected the quality of data in certain national registries. ${ }^{44}$

\section{Complications/outcomes}

Complications and comorbidities of NAFLD are diverse among different ethnicities. The most common cardiovascular complications or comorbidities in China are carotid and coronary artery disease, followed by hypertension. 45 In the USA, African Americans with NAFLD are more likely to have abdominal aorta calcification than non-Hispanic Caucasians, Chinese Americans and Hispanics, with a $41 \%$ higher prevalence. ${ }^{46}$ In a recent meta-analysis of NAFLD patients comparing patients of different ethnicities, there was no statistically significant difference in severity of fibrosis among Hispanics, non-Hispanic Caucasians, and Blacks. Regarding outcomes of NALFD, including progression to cirrhosis, development of HCC, liver-related mortality and all-cause mortality, the data are heterogeneous. ${ }^{47}$ The annual incidence of HCC in Asian countries is higher than the global incidence rate for HCC ( 1.8 cases per 1,000 person-years $v s$. 0.44 case per 1,000 personyears), which may be related to a lack of public awareness of the disease. Consequently, this may lead to delays in proper intervention, monitoring and screening for HCC in these countries. 29,48

Some studies suggest that Hispanics have higher rates of disease progression and fibrosis. ${ }^{30}$ Data analysis from the Scientific Registry of Transplant Recipients between 2002 and 2019 demonstrated that the rate of NASH is lower in Black patients and the majority of liver transplant waitlist candidates for NASH are of Hispanic ethnicity. Moreover, $\mathrm{NASH}-\mathrm{HCC}$ is the leading indication for transplantation in Hispanic patients. 49

\section{Socioeconomic effect}

There is a significant socioeconomic effect on NAFLD. Shifting the pattern of lifestyle and diet have contributed to the prevalence of obesity and NAFLD in Asia. The urbanization of countries in Asia promotes the risks of NAFLD in those populations with increasing prevalence of obesity and DM. The lowest prevalence of disease is in Japan, while the highest rates of NAFLD associated-cirrhosis is in Japanese-Americans, ${ }^{50}$ suggesting the implication of lifestyle and socioeconomic conditions in the prevalence of NAFLD. 48 Similarly, differences in prevalence of NAFLD between Africans in Nigeria and African Americans indicates the implication socioeconomic effect on the epidimology. ${ }^{51}$ The prevalence among the younger Chinese population $(<60$ years-old) was also higher compared to similarly aged patients in the West that is due to rapidly increasing in prevalence of NAFLD from 2008-2010 to 2015-2018 along with rapid change in socioeconomic status. ${ }^{45} \mathrm{~A}$ population-based study in southwest Iran suggested that patients with a high socioeconomic level were likely to have NAFLD. ${ }^{52}$ On the other hand, socioeconomic conditions impact the outcomes of NAFLD. The proportion of deaths from NASH was lowest in the high-income Asia Pacific region. ${ }^{51}$ Poor awareness of NAFLD and less health care utilization in China may contribute to the higher incidence of HCC. ${ }^{45,53,54}$

\section{Risk factors}

\section{Weight}

The World Health Organization estimated in 2016 that there are more than 1.9 billion adults who are considered overweight or obese. ${ }^{29}$ The USA has the highest overall number of obese adults and Indonesia is reported to have the lowest. In terms of prevalence, Oceania has the highest prevalence, while the Middle East is second in prevalence. ${ }^{29} \mathrm{~A}$ recent study demonstrated lower rates of overweight and obese individuals in China, with a higher prevalence and incidence of NAFLD than Western countries. Genetic factors with an involvement in high frequency of NAFLD promotion genes and low frequency of NAFLD risk reduction genes might contribute to the predisposition of NAFLD and metabolic disease in the Chinese population. 45,48 The World Health Organization has classified Asians with BMIs of $23-27.5 \mathrm{~kg} / \mathrm{m}^{2}$ to be at increased risk of obesity-related conditions. ${ }^{55}$

NAFLD in lean individuals, which is commonly defined as NAFLD in patients with a BMI $<25 \mathrm{~kg} / \mathrm{m}^{2}$, has been studied among different ethnicities and nationalities, including Asians, Indians, and Caucasians. The overall prevalence of lean NAFLD is $5-26 \%$ in Asian populations, and $7-20 \%$ in Western populations. These data should be interpreted with caution, given the different BMI cut-offs among different regions. Among Asian countries, the prevalence ranges from approximately $7.27-8 \%$ in China, $4.2 \%$ in Taiwan, $19.3 \%$ in Hong Kong, $12.6-22.4 \%$ in Korea, $15.2 \%$ in Japan, and $6.4 \%$ in India. Compared to Asia, the data in Caucasians are sparse, with smaller sample sizes. A meta-analysis of 93 international studies estimates that $40 \%$ of patients with NAFLD globally are non-obese and an estimated $20 \%$ were classified as lean NAFLD. The prevalence of non-obese NAFLD notably varied quite significantly amongst different countries, with a reported prevalence as low as $25 \%$ in Pakistan to more than half of the general Austrian and Swedish population. ${ }^{56}$ Other country specific studies have reported lean NAFLD rates of $16 \%$ in Italy, $17 \%$ in the Dallas Heart Study, and $7.4 \%$ in the largest epidemiological study in USA. Lean NAFLD is associated with proinflammatory 
visceral adipose tissue, central obesity, higher triglyceride level and early adulthood weight gain in Asian populations, whereas it is associated with insulin resistance, younger age, female sex, and hypercholesterolemia in the Caucasian population. ${ }^{57}$ Another study demonstrated 2- to 3-fold higher risk of insulin resistance in Asian Indians compared to matched Caucasians, Africans, and Eastern Asians. ${ }^{58} \mathrm{~A}$ multiethnic study in the USA also showed that non-obese Hispanics, Chinese Americans, and South Asians were at higher risk of being metabolically ill health compared to non-Hispanic Caucasians due to insulin resistance. 59

In longitudinal studies, development of advanced liver disease in lean NAFLD is associated with hypertriglyceridemia and higher creatinine in Asians. Moreover, lean NAFLD patients in one study were more likely have disease progression compared to obese NAFLD patients amongst Caucasians. ${ }^{57}$ In fact, global estimates of NASH and stage 2 or greater fibrosis in non-obese and lean NAFLD patients are $39.0 \%$ and $29.2 \%$, respectively. Consequently, this has been linked to all-cause, liver-related, and cardiovascularrelated mortality rates of $12.1,4.1$, and 4.0 per 1,000 person-years in this particular demographic. ${ }^{56} \mathrm{~A}$ multicenter study from Italy further classified the risk of disease progression and comorbidities in lean vs. obese patients with NAFLD. In this patient cohort, individuals with lean NAFLD were less likely to have metabolic syndrome and its associated complications, including hypertension, diabetes and carotid artery disease, and less likely to have NASH or advanced fibrosis compared to overweight and obese NAFLD patients. Interestingly, waist circumference appeared to be an important factor in determining the risk of associated comorbidities and disease progression. In fact, lean NAFLD patients with a medium waist circumference (men: 94-102 $\mathrm{cm}$; women: $80-88 \mathrm{~cm}$ ) were more likely to be diabetic than overweight and obese patients with a similar waist circumference. In the overall study population, individuals in the highest waist circumference category were at the highest risk for disease progression, regardless of BMI. 60 Factors that might contribute to lean NAFLD encompasses concomitant alcohol intake, endocrine disorders (polycystic ovarian syndrome, hypothyroidism, growth hormone deficiency), congenital and acquired lipodsystrophy from human immunodeficiency virus medications, association with certain drugs (such as steroids, amiodarone, methotrexate and tamoxifen), inborn errors of metabolism (lysosomal acid lipase deficiency), genetic factors [polymorphisms in the gene that encodes for patatin-like phospholipase domaincontaining protein 3 (PNPLA3)], nutritional factors (starvation, total parental nutrition), and gastrointestinal surgery (jejunoileal bypass). ${ }^{61}$

\section{Metabolic syndrome}

Metabolic syndrome (MS) includes abdominal obesity, DM, hypertension, and dyslipidemia.62 Both obesity and DM are increasing globally and are correlated with increased prevalence of NAFLD. Type 2 DM is a major risk factor for the development of NAFLD and accelerates progression to advanced liver disease and increase risk for mortality. According to the World Health Organization, there has been an increase in DM from 108 million in 1980 to 422 million in 2014, with a notable increase amongst patients in low and middle income countries. ${ }^{63}$ The prevalence of NAFLD/ NASH among type 2 DM is over $60 \% .{ }^{64}$ Similarly, the prevalence is $52.55 \%$ in Asians. ${ }^{48}$ In the USA, Hispanics have a $12.8 \%$, non-Hispanic Blacks have $13.2 \%$ and Asian Americans have $9 \%$ higher risk of type 2 DM, respectively, when compared to non-Hispanic Caucasians. ${ }^{65}$ Native American adults in Southern Arizona have one of the highest preva- lence rates of DM in the world, estimated at 33\%. According to the 2019 International Diabetes Atlas, the prevalence of diabetes in adults was $8.5 \%$ in South and Central America, $12.2 \%$ in the Middle East and North Africa, $4.7 \%$ in Africa, $11.5 \%$ in South-East Asia, $11.4 \%$ in the Western Pacific, and $6.3 \%$ in Europe. ${ }^{65}$

Hypertension is another risk factor for NAFLD and has a bidirectional relationship with NAFLD. The severity and progression of NAFLD have been associated with hypertension. On the other hand, NAFLD is also a possible risk factor for development of hypertension. 66 In the USA, the prevalence of hypertension is higher in non-Hispanic Blacks compared to non-Hispanic White and Hispanic populations. ${ }^{67}$ When the 2017 American College of Cardiology/ American Heart Association hypertension guideline was applied globally, the prevalence of hypertension increased in Canada $(32 \%$ to $46 \%), 68$ India (29\% to $43 \%),{ }^{69}$ China ( $25 \%$ to $\left.50 \%\right),{ }^{70}$ and South Korea $(28 \%$ to $48 \%), 71$ respectively.

Dyslipidemia induces inflammation and increases cytokine production and oxidative stress, triggering pathogenesis of NAFLD. Moreover, dyslipidemia is responsible for cardiovascular risk in NAFLD patients. ${ }^{72}$ Hypertriglyceridemia was found to be highest in East Asians and non-Hispanic Whites and lowest in the African American population. ${ }^{73}$ The prevalence of high low-density lipoprotein cholesterol was higher in Asian Indians, Filipinos, Japanese, and Vietnamese compared to non-Hispanic Whites. ${ }^{74}$ MS was found to be present in $34 \%, 62 \%, 31 \%, 33 \%$, and $37 \%$ of patients with NAFLD in Asia, Europe, Middle East, North America and South America, respectively. The incidence of MS is higher among NAFLD patients compared to controls. In Asia, the incidence increases by $14 \%$ if patients have at least three components of MS compared to patients with fewer MS. ${ }^{5}$ NAFLD patients with multiple components of MS are at higher risk for advanced fibrosis and all-cause mortality. Each additional MS condition worsens the risk of liverrelated and all-cause mortality based on The Third National Health and Nutrition Examination Survey (commonly known as NHANES). ${ }^{75}$

\section{Genetics}

With the advent of genome-wide association studies (commonly referred to as GWAS), many genes have been brought to the forefront of NAFLD research. A select few of these genes have been further explored with respect to their role in NAFLD development in different ethnicities, and many more are under investigation.

One of the most notable genes in the field of NAFLD codes for PNPLA3. In particular, the genetic variant rs738409, which results in substitution of methionine for isoleucine, resulting in a loss of function in the PNPLA3 protein, contributes to increased accumulation of triglycerides in lipid droplets within hepatocytes as compared to cells with functional PNPLA3. ${ }^{76}$ This variant has been associated with increased susceptibility to NAFLD in a variety of different ethnicities, including Hispanics, African Americans, East Asians, and South Asians. Particularly in studies from Asia, a "lean NAFLD" phenotype, as previously mentioned, is well described. These patients have a BMI that is lower than the commonly accepted obese range, and yet still develop NAFLD at notable rates. ${ }^{77}$ A study from Hong Kong noted that the rs738409 allele is more common in patients with "lean NAFLD" than in obese patients. ${ }^{78}$ Another gene variant of PNPLA3, rs6006460, results in a phenotype characterized by lower than average hepatic fat. This variant, interestingly, occurs in $10 \%$ of African Americans as opposed to $<1 \%$ in either Caucasians or Hispanics, ${ }^{79}$ and may in part explain the lower observed incidence of NAFLD amongst this 
patient demographic.

Another gene linked to the development of NAFLD that has also been studied within the context of ethnicity codes for the membrane-bound o-acyltransferase domain-containing 7 protein. The rs641738 variant has been associated with increased NAFLD risk in Europeans ${ }^{80}$ but has not been studied in other populations as extensively. Two other genes with variants associated with NAFLD, TM6SF2 and GCKR, have been well described. TM6SF2 codes for a protein of unknown function, although different variants have been linked to increased hepatic lipid content, as well as increased levels of aspartate aminotransferase/alanine aminotranferase. ${ }^{81}$ GCKR, which codes for a glucokinase regulator, similarly has variants that are associated with hepatic fat accumulation. ${ }^{82}$ A population-based study among Hispanic/Latino adults in the USA found a high frequency of PNPLA3 G (41\%) and a low frequency of TM6SF2 T (5\%) in Hispanics/Latinos. Among Hispanics, the PNPLA3 G frequency was the highest in Mexicans (52\%) and the lowest in Dominicans (23\%). ${ }^{83}$ In another study that compared genetic predisposition between Chinese and Caucasians, the frequency of PNPLA3 polymorphism was higher and that of TM6SF2 was lower in Chinese compared to Caucasians. 45

GWAS studies have also yielded a number of different genes associated with NAFLD, including but not limited to LPIN1, Tribble-1, FDFT1, ERLIN1, etc. Genes involved in the pathophysiology of other liver conditions have also been noted to contribute to risk of NAFLD development; for example, mutations in $H F E$, most commonly noted in hereditary hemochromatosis, have been cited as risk factors for NAFLD. ${ }^{84}$ It is also worth mentioning that ongoing investigations into epigenetics, microRNA, and mitochondrial RNA may improve our understanding of how NAFLD presents in different ethnicities in the future.

\section{Lifestyle}

\section{Diet}

Although it seems intuitive that certain elements of diet and weight control would predispose specific populations to NAFLD, the literature that explores these factors is more recent. Overall, increased intake of saturated fats, fructose, and cholesterol-rich sources predisposes individuals to NAFLD. ${ }^{85}$ Although evidence currently is limited, sedentary behavior has been increasingly accepted as an independent risk factor for NAFLD. ${ }^{86}$ As an example to the beneficial effect of physical activity, aerobic exercise that contributes to progressive increase in fat-free, lean mass can provide protection against NAFLD. Dietary elements typical of the Western Diet in addition to Western eating habits, such as snacking, have been shown to independently contribute to hepatic steatosis. ${ }^{87}$ Soft-drink consumption, rampant in the West, has been shown to increase liver fat by $140 \%$ over a period of 6 months in otherwise healthy individuals. 88 Relatedly, it was found that a diet of $3 \mathrm{~g}$ fructose $/ \mathrm{kg}$ increases the amount of hepatic fat in adult men. 89

Limiting consumption of carbohydrates has generally been noted to improve NAFLD; 90 however, if carbohydrates are examined overall, a focus on ethnic diet variations presents a more complicated picture. For example, the traditional Chinese diet is high in carbohydrates but is also vegetable-rich and has proven to be low-risk for NAFLD. Consistent with earlier mentioned data, a more recent study noted that "Westernization" of South Korean food, via refined grains, processed meats, fried foods, etc., correlated with increased incidence of NAFLD diagnosed by ultrasound. ${ }^{91}$ Such regions have also noted that weight gain in general, irrespective of diet, seems to predispose people to NAFLD in certain regional studies. Korean studies have noted that weight gain as low as $2 \mathrm{~kg}$ can contribute to NAFLD development.92 More specifically, data from Hong Kong reported that the presence of central obesity predisposes patients to NAFLD. ${ }^{93}$

Variations and similarities in the standard diet within a geographic region or amongst individuals of a particular ethnic background has allowed researchers to investigate the different nutritional patterns that promote hepatic steatosis and the progression of NAFLD, as well as whether a specific diet affects disease progression differently in patients of various backgrounds. Ultimately, this informs healthcare providers when counseling patients with NAFLD on ideal eating habits, and whether recommendations can be generalizable to individuals of various ethnicities.

The Western dietary pattern, containing large amounts of red meat, processed meat, and fried foods, has a wellestablished link to the development of MS. ${ }^{94}$ Unfortunately, this diet has established itself and its associated adverse health consequences globally. A study of 170 Iranians with NAFLD evaluated the effects of an Iranian, Western and "healthy dietary patterns" on liver fibrosis. The Western dietary pattern was strongly associated with fibrosis, with an odds ratio of 4.21. The investigators noted that higher consumption of red meat, hydrogenated fats, and soda drinks increased the odds of fibrosis measured by elastography, while a diet rich in low-fat diary, nuts, fruit, and coffee or tea was protective. ${ }^{95}$ Interestingly, the positive association between a Western diet and NAFLD was not replicated in a prospective cross-sectional study of 1,190 Korean patients with and without NAFLD. Four dietary patterns and their association with a diagnosis of hepatic steatosis were analyzed, including a traditional Korean diet, Western and high-carbohydrate diets, and a simple meal pattern diet. As previously mentioned, this study revealed no association between a Western or carbohydrate-rich diet and the presence of NAFLD, while a traditional Korean diet was positively correlated with presence of the disease. ${ }^{96}$ While the absence of an association between increased carbohydrate intake and NAFLD is somewhat surprising, data from other studies have been inconsistent with regards to the effect of carbohydrate intake. Patients who were on a 2-week carbohydrate-restricted diet had similar weight loss but more hepatic fat reduction than patients who were on a reduced calorie-only diet. 90 However, another study demonstrated similar improvements in hepatic steatosis between patients on a high-carbohydrate diet and low-carbohydrate diet when weight loss was comparable, ${ }^{97}$ suggesting that the true benefit of carbohydrate restriction on NAFLD arises primarily when it is linked to weight reduction. However, a low carbohydrate diet in patients with NAFLD has been associated with a decrease in alanine aminotransferase, although the diet was primarily soy-based. ${ }^{98}$

In contrast to the Korean dietary study and similar to findings of the Iranian investigators, a study out of Greece confirmed the increased odds of NAFLD with a fast-food type dietary pattern, while also confirming that when unsaturated fatty acid intake was divide into quartiles, those in the second quartile had an over $50 \%$ reduced odds of NAFLD compared to individuals within the first quartile of dietary intake. ${ }^{99}$ With respect to fats, a potential therapeutic strategy includes increased consumption of both mono-unsaturated fatty acids and poly-unsaturated fatty acids. Increased intake of poly-unsaturated fatty acids results in greater reduction in hepatic steatosis when used in combination with a heart healthy diet compared to dieting alone. ${ }^{100}$ In fact, a meta-analysis has found that omega-3 fatty acids derived from seafood sources have a positive effect on hepatic steatosis. ${ }^{101}$

Given the increased popularity and presence globally of the Western style diet, the above data generally seem to 
Han M.A.T. et al: NAFLD in different ethnicities

suggest a benefit to avoiding this type of nutritional behavior. In fact, a study assessing patients (Framingham Heart Study), consisting primarily of Caucasian patients in the USA reinforced the benefits of focusing on alternative diets for liver fat accumulation. The study investigated how changes in the Mediterranean-style diet score and Alternative Healthy Eating Index affected liver fat and new-onset fatty liver. An increase in either dietary score was inversely associated with liver fat accumulation and incident NAFLD, with a reduction in the odds of fatty liver by $26 \%$ for every 1 -standard deviation increase in Mediterranean-style diet score. Moreover, individuals with a higher genetic predisposition to NAFLD as determined by single nucleotide polymorphisms and decreased Mediterranean-style diet score or Alternative Healthy Eating Index scores had higher liver fat compared to patients with improved or stable scores. ${ }^{102}$ Thus, adopting the Mediterranean diet, typically characterized by high intake of olive oil, nuts, fruits, vegetables, legumes, and fish, with wine in moderation, can be suggested to patients with NAFLD, especially given the broad health benefits related to a variety of different health conditions related to MS. Despite heterogeneity in the way the Mediterranean Diet is defined in different studies, it has consistently shown favorable health outcomes. ${ }^{103}$ Studies from multiple regions of the world have reported a marked regression of NAFLD when patients switched to the Mediterranean Diet, ${ }^{104}$ reinforcing its broad appeal regardless of patient ethnicity. A large randomized control trial to evaluate the benefit of this diet, independent of weight loss, is currently underway in Australia. ${ }^{105}$

With respect to protein intake, it seems that a diet consisting of a larger proportion of protein does not necessarily aid in improvement of NAFLD. However, a moderate protein diet encompassing $25 \%$ of total caloric intake has been shown to be optimal, and higher percentages do not necessarily reduce body fat content any better. ${ }^{106}$

Ultimately, the guidance provided by a patient's healthcare provider is key to successful changes in dietary habits that can ultimately improve or reverse hepatic steatosis. Studies show that patients will make better nutritional choices after having expressed better understanding of what NAFLD is, reinforcing the importance of patient education. Moreover, as a preventative measure, recommending dietary patterns that reflect an adherence to a healthy diet can reduce NAFLD risk in the general population. An analysis of the multiethnic cohort consisting of patients of African American, Japanese American, Latino, Native Hawaiian, and Caucasian descent revealed that high Healthy Eating Index and Dietary Approaches to Stop Hypertension scores were associated with lower risk of fatty liver, with no observed differences by race or ethnicity. ${ }^{107}$ However, a more recent analysis of the same cohort focused on the specific components of enrollees' diets at baseline and association with NAFLD. Overall, when comparing 2,974 patients with NAFLD and 29,474 matched controls, intake of poultry, cholesterol, processed red meat and red meat in general was associated with NAFLD. When stratified by race and ethnicity, poultry intake and cholesterol intake was only significantly associated with NAFLD in Whites and Native Hawaiians. Processed red meat correlated significantly with NAFLD in Latinos and Whites, and increased fiber intake was protective in these two ethnic groups. ${ }^{108}$ The damaging effects of red meat consumption and the associated risk of NAFLD was further demonstrated in a cross-sectional study of 789 adults. After controlling for BMI, smoking, alcohol intake, physical activity, energy and saturated fat and cholesterol intake, the consumption of meat in general and red or processed meat was associated with increased odds for insulin resistance and NAFLD. ${ }^{109}$ Thus, while an overall healthy diet is recommended for all patients, with the ultimate goal of achieving meaningful weight loss, a specific list of foods to avoid may be tailored to a patient based on race and ethnicity.

Intermittent fasting has recently gained popularity as an alternative to more traditional diets, aimed at reducing weight and improving obesity-related comorbidities. It includes diets that focus on a particular eating pattern where caloric intake is eliminated during a predefined period of time, and has consistently proven beneficial for both weight loss and reduction in obesity-related comorbidities. ${ }^{110} \mathrm{~A}$ randomized controlled trial assessing a modified alternative-day calorie restriction diet, another form of intermittent fasting, found that adherence to this diet amongst patients with NAFLD was excellent (75-83\%) with a significant reduction in liver steatosis and fibrosis (measured by shear wave elastography) amongst individuals randomized to 8 weeks of the intervention diet arm.111

\section{Physical activity}

Exercise as an intervention in NAFLD has also been studied, although the data concerning its benefits independent of the weight loss requires further clarification. Studies have noted a 20-30\% decrease in hepatic lipid content with general exercise. Interestingly, even when these patients regain lost weight, there seems to be a persistent long lasting beneficial effect on liver fat and insulin resistance. ${ }^{112}$ Aerobic exercise has been shown to reduce hepatic triglycerides in sedentary and obese patients. ${ }^{113}$ Weight resistance exercise has also been linked to a reduction in hepatic steatosis without weight loss. ${ }^{114}$ Although evidence on aerobic vs. resistance training is mixed, combination therapy seems to be superior to either. However, weight loss was a confounder when comparing aerobic with resistance exercise and a combination of the two, 115 and thus these recommendations require further investigation prior to unlinking their benefits to weight loss alone.

Non-obese patients with underlying NAFLD or NASH can be more challenging to manage in the absence of approved pharmacotherapy. As mentioned previously, the strongest evidence for the management of NAFLD and NASH comes from studies focused on interventions that achieve weight loss and increased physical activity. While implementing these interventions intuitively makes sense in obese individuals or those with other components of MS, it is less obvious for those considered within normal BMI range, which has been more commonly seen in patients of east Asian background. ${ }^{116}$ However, a study examining the effect of diet modification and exercise on hepatic steatosis in 1,365 potential living donors with NAFLD on initial biopsy revealed that although only $5 \%$ of patients were obese at the start of the study, histological improvement on repeat biopsy was observed in $85.8 \%$ of participants. ${ }^{117}$ Some of these findings may, in part, be related to the response to weight loss seen with variants of the PNPLA3 alleles. More specifically, the GG genotype that is more commonly observed in Asian patients with "lean NAFLD" has been linked to a favorable histologic response to diet modification leading to weight loss. Thus, despite the challenges associated with recommending weight loss to patients with a normal BMI, interventions that reduce weight are likely to be beneficial in this patient population. Moreover, although "lean NAFLD" is well recognized in patients of Asian background, with prevalence as high as $19 \%$ in Asia compared to $7 \%$ in the USA, this phenotype is now increasingly recognized in other races. In fact, a large Swedish cohort with 646 patients with biopsy-proven NAFLD, the "lean NAFLD" prevalence was reported as $19 \%, 118$ identical to estimates from Asia, making these recommendations more generalizable than previously thought.

Studies assessing the efficacy of caffeinated beverages 
have shown consistent results. Overall, while the majority of studies do not show any significant improvement in steatosis with increased caffeine intake, coffee and other caffeinated beverages may have a protective effect against the development or presence of fibrosis. ${ }^{119}$ An analysis of the multiethnic cohort evaluating the association between coffee intake and chronic liver disease and HCC revealed an inverse association between increased coffee consumption and the incidence of HCC or chronic liver disease. In fact, consuming $\geq 4$ cups of coffee a day was associated with a $41 \%$ reduction in $\mathrm{HCC}$ and a $71 \%$ reduction in chronic liver disease, when compared to non-coffee drinkers. This association did not differ based on patient race or ethnicity. Given the risk of HCC associated with NAFLD, including in the absence of cirrhosis, a balanced increase in coffee intake may be beneficial regardless of race. ${ }^{120}$

\section{Conclusions}

The prevalence of NAFLD varies globally. Different ethnicities carry distinct risks for NAFLD. The shifting paradigm of socioeconomic status, lifestyle, and dietary habits plays an important role in raising the incidence of NAFLD irrespective of genetic and geographic background. Those changes are diverse among different ethnicities and/or countries. The disparity in availability and accessibility to certain diagnostic tests and healthcare utilization in different countries also impacts the rates of outcomes or complications. We have briefly summarized the characteristics of NAFLD in different ethnic populations in Table 1. While it is important to develop universal guidelines that can be used for patients of any ethnic background, it is also imperative that diagnosis and management of NAFLD is tailored differently amongst various populations. We need more effort in educating patients on NAFLD and improvement in the utilization of noninvasive tests to risk-stratify these patients. Obtainment of more data on socioeconomic effects of NAFLD is necessary, as such plays a major role in development and complications of NAFLD. Finally, it is important for nations to work together to implement education and prevention programs to decrease the growing burden from this disease.

\section{Funding}

None to declare.

\section{Conflict of interest}

The authors have no conflict of interests related to this publication.

\section{Author contributions}

Drafting of manuscript (QY), critical revision of the manuscript for the important intellectual content (MATH, ZT, NP), administrative support (NP), supervision (NP).

\section{References}

[1] George J, Anstee Q, Ratziu V, Sanyal A. NAFLD: The evolving landscape. J Hepatol 2018;68:227-229. doi:10.1016/j.jhep.2017.11.016.

[2] Feldman M, Friedman LS, Brandt LJ. Sleisenger and Fordtran's gastrointestinal and liver disease: Pathophysiology/diagnosis/management. Philadelphia, PA. Saunders/Elsevier; 2016.

[3] Eslam M, Newsome PN, Sarin SK, Anstee QM, Targher G, Romero-Gomez $M$, et al. A new definition for metabolic dysfunction-associated fatty liver 
disease: An international expert consensus statement. J Hepatol 2020;73: 202-209. doi:10.1016/j.jhep.2020.03.039.

[4] Eslam M, Sanyal AJ, George J. MAFLD: A consensus-driven proposed nomenclature for metabolic associated fatty liver disease. Gastroenterology 2020;158:1999-2014.e1. doi:10.1053/j.gastro.2019.11.312.

[5] Younossi Z, Tacke F, Arrese M, Chander Sharma B, Mostafa I, et al. Global perspectives on nonalcoholic fatty liver disease and nonalcoholic steatohepatitis. Hepatology 2019;69:2672-2682. doi:10.1002/hep.30251.

[6] Vos MB, Abrams SH, Barlow SE, Caprio S, Daniels SR, Kohli R, et al. NASPGHAN clinical practice guideline for the diagnosis and treatment of nonalcoholic fatty liver disease in children: Recommendations from the Expert Committee on NAFLD (ECON) and the North American Society of Pediatric Gastroenterology, Hepatology and Nutrition (NASPGHAN). J Pediatr Gastroenterol Nutr 2017;64:319-334. doi:10.1097/MPG.0000000000001482.

[7] Chalasani N, Younossi Z, Lavine JE, Charlton M, Cusi K, Rinella M, et al. The diagnosis and management of nonalcoholic fatty liver disease: Practice guidance from the American Association for the Study of Liver Diseases. Hepatology 2018;67:328-357. doi:10.1002/hep.29367.

[8] Anstee OM, Reeves HL, Kotsiliti E, Govaere O, Heikenwalder M. From NASH to HCC: current concepts and future challenges. Nat Rev Gastroenterol Hepatol 2019;16:411-428. doi:10.1038/s41575-019-0145-7.

[9] Huber Y, Boyle M, Hallsworth K, Tiniakos D, Straub BK, Labenz C, et al. Health-related quality of life in nonalcoholic fatty liver disease associates with hepatic inflammation. Clin Gastroenterol Hepatol 2019;17:20852092.e1. doi:10.1016/j.cgh.2018.12.016.

[10] Yamamura S, Nakano D, Hashida R, Tsutsumi T, Kawaguchi T, Okada M, et al. Patient-reported outcomes in patients with non-alcoholic fatty liver disease: A narrative review of Chronic Liver Disease Questionnaire-nonalcoholic fatty liver disease/non-alcoholic steatohepatitis. J Gastroenterol Hepatol 2020. doi:10.1111/jgh.15172.

[11] Younossi ZM, Wong VW, Anstee QM, Romero-Gomez M, Trauner MH, Harrison $\mathrm{SA}$, et al. Fatigue and pruritus in patients with advanced fibrosis due to nonalcoholic steatohepatitis: The impact on patient-reported outcomes. Hepatol Commun 2020;4:1637-1650. doi:10.1002/hep4.1581.

[12] Geier A, Rinella M, Balp MM, McKenna SJ, Brass C, Przybysz R, et al. Realworld burden of nonalcoholic steatohepatitis. Clin Gastroenterol Hepatol 2020. doi:10.1016/j.cgh.2020.06.064.

[13] Dasarathy S, Dasarathy J, Khiyami A, Joseph R, Lopez R, McCullough AJ. Validity of real time ultrasound in the diagnosis of hepatic steatosis: a prospective study. J Hepatol 2009;51:1061-1067. doi:10.1016/j.jhep. 2009.09.001.

[14] Bedogni G, Bellentani S, Miglioli L, Masutti F, Passalacqua M, Castiglione $A$, et al. The Fatty Liver Index: a simple and accurate predictor of hepatic steatosis in the general population. BMC Gastroenterol 2006;6:33. doi:10.1186/1471-230X-6-33.

[15] Lee JH, Kim D, Kim HJ, Lee CH, Yang JI, Kim W, et al. Hepatic steatosis index: a simple screening tool reflecting nonalcoholic fatty liver disease. index: a simple screening tool reflecting nonalcoholic fatty liver

[16] Ashtari S, Vahedi M, Amin Pourhoseingholi M, Reza Zali M. Evaluated outcomes in patients with Chronic Hepatitis C. Gastroenterol Hepatol Bed Bench. 2013;6:S58-S64.

[17] Wong VW, Petta S, Hiriart JB, Cammà C, Wong GL, Marra F, et al. Validity criteria for the diagnosis of fatty liver by $\mathrm{M}$ probe-based controlled attenuation parameter. J Hepatol 2017;67:577-584. doi:10.1016/j.jhep.2017.05.005.

[18] Wong VW, Chu WC, Wong GL, Chan RS, Chim AM, Ong A, et al. Prevalence of non-alcoholic fatty liver disease and advanced fibrosis in Hong Kong Chinese: a population study using proton-magnetic resonance spectroscopy and transient elastography. Gut 2012;61:409-415. doi:10.1136/ gutjnl-2011-300342.

[19] Byrne CD, Patel J, Scorletti E, Targher G. Tests for diagnosing and monitoring non-alcoholic fatty liver disease in adults. BMJ 2018;362:k2734. doi:10.1136/bmj.k2734

[20] Kotronen A, Peltonen M, Hakkarainen A, Sevastianova K, Bergholm R, Johansson LM, et al. Prediction of non-alcoholic fatty liver disease and liver fat using metabolic and genetic factors. Gastroenterology 2009;137:865fat using metabolic and genetic factors.
872. doi:10.1053/j.gastro.2009.06.005.

[21] Vilar-Gomez E, Calzadilla-Bertot L, Wai-Sun Wong V, Castellanos M, Allerde la Fuente R, Metwally $M$, et al. Fibrosis severity as a determinant of cause-specific mortality in patients with advanced nonalcoholic fatty liver disease: A multi-national cohort study. Gastroenterology 2018;155:443457.e17. doi:10.1053/j.gastro.2018.04.034.

[22] Han MAT. Noninvasive tests (NITs) for hepatic fibrosis in fatty liver syndrome. Life (Basel) 2020;10:198. doi:10.3390/life10090198.

[23] Samji NS, Snell PD, Singal AK, Satapathy SK. Racial disparities in diagnosis and prognosis of nonalcoholic fatty liver disease. Clin Liver Dis (Hoboken) 2020;16:66-72. doi:10.1002/cld.948.

[24] Friedrich-Rust M, Romen D, Vermehren J, Kriener S, Sadet D, Herrmann E, et al. Acoustic radiation force impulse-imaging and transient elastography for non-invasive assessment of liver fibrosis and steatosis in NAFLD. Eur J Radiol 2012;81:e325-e331. doi:10.1016/j.ejrad.2011.10.029.

[25] Caussy C, Chen J, Alquiraish MH, Cepin S, Nguyen P, Hernandez C, et al. Association between obesity and discordance in fibrosis stage determination by magnetic resonance vs transient elastography in patients with nonalcoholic liver disease. Clin Gastroenterol Hepatol 2018;16:1974-1982.e7. alcoholic liver disease. Clin Gastr.

[26] Xiao G, Zhu S, Xiao X, Yan L, Yang J, Wu G. Comparison of laboratory tests, ultrasound, or magnetic resonance elastography to detect fibrosis in patients with nonalcoholic fatty liver disease: A meta-analysis. Hepatology 2017;66:1486-1501. doi:10.1002/hep.29302.

[27] Younossi ZM, Koenig AB, Abdelatif D, Fazel Y, Henry L, Wymer M. Global epidemiology of nonalcoholic fatty liver disease-Meta-analytic assessment of prevalence, incidence, and outcomes. Hepatology 2016;64:73-84. doi:10.1002/hep.28431.

[28] Torres DM, Williams CD, Harrison SA. Features, diagnosis, and treatment of nonalcoholic fatty liver disease. Clin Gastroenterol Hepatol 2012;10:837858. doi:10.1016/j.cgh.2012.03.011.

[29] Younossi ZM. Non-alcoholic fatty liver disease - A global public health perspective. J Hepatol 2019;70:531-544. doi:10.1016/j.jhep.2018.10.033.

[30] Browning JD, Szczepaniak LS, Dobbins R, Nuremberg P, Horton JD, Cohen $\mathrm{JC}$, et al. Prevalence of hepatic steatosis in an urban population in the United States: impact of ethnicity. Hepatology 2004;40:1387-1395. the United States: impact

[31] Lim U, Monroe KR, Buchthal S, Fan B, Cheng I, Kristal BS, et al. Propensity for intra-abdominal and hepatic adiposity varies among ethnic groups. Gastroenterology 2019;156:966-975.e10. doi:10.1053/j.gastro.2018.11.021.

[32] Fischer GE, Bialek SP, Homan CE, Livingston SE, McMahon BJ. Chronic liver disease among Alaska-Native people, 2003-2004. Am J Gastroenterol 2009;104:363-370. doi:10.1038/ajg.2008.57.

[33] Fleischman MW, Budoff M, Zeb I, Li D, Foster T. NAFLD prevalence differs among hispanic subgroups: the Multi-Ethnic Study of Atherosclero-
sis. World J Gastroenterol 2014;20:4987-4993. doi:10.3748/wjg.v20. sis. World

[34] Kallwitz ER, Daviglus ML, Allison MA, Emory KT, Zhao L, Kuniholm MH, et al. Prevalence of suspected nonalcoholic fatty liver disease in Hispanic/Latino individuals differs by heritage. Clin Gastroenterol Hepatol 2015;13:569576. doi:10.1016/j.cgh.2014.08.037.

[35] Karnikowski M, Córdova C, Oliveira RJ, Karnikowski MG, Nóbrega Ode T. Non-alcoholic fatty liver disease and metabolic syndrome in Brazilian middle-aged and older adults. Sao Paulo Med J 2007;125:333-337. doi:10.1590/s1516-31802007000600006.

[36] Messiah SE, Vidot DC, Bispo JB, Arheart KL, Khorgami Z, Cruz-Muñoz NDL. Ethnic and gender differences in the prevalence of nonalcoholic steatohepatitis among bariatric surgery patients. Bariatric Surgical Practice and Patient Care 2016;11:153-157. doi:10.1089/bari.2016.0018.

[37] Rinella ME. Nonalcoholic fatty liver disease: a systematic review. JAMA 2015;313:2263-2273. doi:10.1001/jama.2015.5370.

[38] Radu C, Grigorescu M, Crisan D, Lupsor M, Constantin D, Dina L. Prevalence and associated risk factors of non-alcoholic fatty liver disease in hospitalized patients. J Gastrointestin Liver Dis 2008;17:255-260.

[39] Alazawi W, Mathur R, Abeysekera K, Hull S, Boomla K, Robson J, et al. Ethnicity and the diagnosis gap in liver disease: a population-based study. $\mathrm{Br}$ J Gen Pract 2014;64:e694-e702. doi:10.3399/bjgp14X682273.

[40] Onyekwere CA, Ogbera AO, Balogun BO. Non-alcoholic fatty liver disease and the metabolic syndrome in an urban hospital serving an African community. Ann Hepatol 2011;10:119-124. doi:10.1016/S16652681(19)31559-5.

[41] Adejumo AC, Samuel GO, Adegbala OM, Adejumo KL, Ojelabi O, Akanbi $\mathrm{O}$, et al. Prevalence, trends, outcomes, and disparities in hospitalizations for nonalcoholic fatty liver disease in the United States. Ann Gastroenterol 2019;32:504-513. doi:10.20524/aog.2019.0402.

[42] Kruger FC, Daniels C, Kidd M, Swart G, Brundyn K, Van Rensburg C, et al. Non-alcoholic fatty liver disease (NAFLD) in the Western Cape: a descriptive analysis. S Afr Med J 2010;100:168-171. doi:10.7196/samj.1422.

[43] Younossi ZM, Blissett D, Blissett R, Henry L, Stepanova M, Younossi Y, et al. The economic and clinical burden of nonalcoholic fatty liver disease in the United States and Europe. Hepatology 2016;64:1577-1586. doi:10.1002/ hep. 28785.

[44] Maziak W. Health in the middle East. BM] 2006;333:815-816. doi:10.1136/ bmj.39006.478796.80.

[45] Zhou J, Zhou F, Wang W, Zhang XJ, Ji YX, Zhang P, et al. Epidemiological features of NAFLD from 1999 to 2018 in China. Hepatology 2020;71:18511864. doi:10.1002/hep.31150.

[46] Remigio-Baker RA, Allison MA, Forbang NI, Loomba R, Anderson CAM, Budoff $M$, et al. Race/ethnic and sex disparities in the non-alcoholic fatty liver disease-abdominal aortic calcification association: The Multi-Ethnic Study of Atherosclerosis. Atherosclerosis 2017;258:89-96. doi:10.1016/j. atherosclerosis.2016.11.021.

[47] Rich NE, Oji S, Mufti AR, Browning JD, Parikh ND, Odewole M, et al. Racial and ethnic disparities in nonalcoholic fatty liver disease prevalence, severity, and outcomes in the United States: A systematic review and metaanalysis. Clin Gastroenterol Hepatol 2018;16:198-210.e2. doi:10.1016/j. cgh.2017.09.041.

[48] Li J, Zou B, Yeo YH, Feng Y, Xie X, Lee DH, et al. Prevalence, incidence, and outcome of non-alcoholic fatty liver disease in Asia, 1999-2019: a systematic review and meta-analysis. Lancet Gastroenterol Hepatol 2019;4:389398. doi:10.1016/S2468-1253(19)30039-1.

[49] Younossi ZM, Stepanova M, Ong J, Trimble G, AlQahtani S, Younossi I, et al. Nonalcoholic steatohepatitis is the most rapidly increasing indication for liver transplantation in the United States. Clin Gastroenterol Hepatol 2020. doi: $10.1016 / \mathrm{j} . \mathrm{cgh} .2020 .05 .064$

[50] Setiawan VW, Stram DO, Porcel ], Lu SC, Le Marchand L, Noureddin M. Prevalence of chronic liver disease and cirrhosis by underlying cause in understudied ethnic groups: The multiethnic cohort. Hepatology 2016;64:1969-1977. doi:10.1002/hep.28677.

[51] The global, regional, and national burden of cirrhosis by cause in 195 countries and territories, 1990-2017: a systematic analysis for the Global Burden of Disease Study 2017. Lancet Gastroenterol Hepatol 2020;5:245266. doi:10.1016/S2468-1253(19)30349-8.

[52] Zarean E, Goujani R, Rahimian G, Ahamdi A. Prevalence and risk factors of non-alcoholic fatty liver disease in southwest Iran: a population-based case-control study. Clin Exp Hepatol 2019;5:224-231. doi:10.5114/ ceh.2019.87635. 
[53] Zhang W, Chao S, Chen S, Rao H, Huang R, Wei L, et al. Awareness and knowledge of nonalcoholic fatty liver disease among office employees in Beijing, China. Dig Dis Sci 2019;64:708-717. doi:10.1007/s10620-0185389-5.

[54] Chen S, Chao S, Konerman M, Zhang W, Rao H, Wu E, et al. Survey of nonalcoholic fatty liver disease knowledge, nutrition, and physical activity patterns among the general public in Beijing, China. Dig Dis Sci 2019;64:3480-3488. doi:10.1007/s10620-019-05709-0.

[55] Appropriate body-mass index for Asian populations and its implications for policy and intervention strategies. Lancet 2004;363:157-163. doi:10.1016/S0140-6736(03)15268-3.

[56] Ye Q, Zou B, Yeo YH, Li J, Huang DQ, Wu Y, et al. Global prevalence, in cidence, and outcomes of non-obese or lean non-alcoholic fatty liver disease: a systematic review and meta-analysis. Lancet Gastroenterol Hepatol 2020;5:739-752. doi:10.1016/S2468-1253(20)30077-7.

[57] Younes R, Bugianesi E. NASH in lean individuals. Semin Liver Dis 2019;39:86-95. doi:10.1055/s-0038-1677517.

[58] Petersen KF, Dufour S, Feng J, Befroy D, Dziura J, Dalla Man C, et al. Increased prevalence of insulin resistance and nonalcoholic fatty liver disease in Asian-Indian men. Proc Natl
doi: $10.1073 /$ pnas.0608537103.

[59] Gujral UP, Vittinghoff E, Mongraw-Chaffin M, Vaidya D, Kandula NR, Allison $M$, et al. Cardiometabolic abnormalities among normal-weight persons from five racial/ethnic groups in the United States: A cross-sectional analysis of two cohort studies. Ann Intern Med 2017;166:628-636. doi:10.7326/M161895.

[60] Fracanzani AL, Petta S, Lombardi R, Pisano G, Russello M, Consonni $D$, et al. Liver and cardiovascular damage in patients with lean nonalcoholic fatty liver disease, and association with visceral obesity. Clin Gastroenterol Hepatol 2017;15:1604-1611.e1. doi:10.1016/j.cgh.2017.04.045.

[61] Younossi ZM, Stepanova M, Negro F, Hallaji S, Younossi Y, Lam B, et al. Nonalcoholic fatty liver disease in lean individuals in the United States. Medicine (Baltimore) 2012;91:319-327. doi:10.1097/MD.0b013e3182779d49.

[62] Grundy SM, Cleeman JI, Daniels SR, Donato KA, Eckel RH, Franklin BA, et al. Diagnosis and management of the metabolic syndrome: an American Heart Association/National Heart, Lung, and Blood Institute Scientific Statement. Circulation 2005;112:2735-2752. doi:10.1161/CIRCULATIONAHA.105.169404.

[63] World Health Organization. Diabetes. Available from: https://www.who. int/health-topics/diabetes\#tab=tab_1

[64] Younossi Z, Anstee QM, Marietti M, Hardy T, Henry L, Eslam M, et al. Global burden of NAFLD and NASH: trends, predictions, risk factors and prevention. Nat Rev Gastroenterol Hepatol 2018;15:11-20. doi:10.1038/nrgastro.2017.109.

[65] International Diabetes Federation. IDF diabetes atlas, 9th edition 2019. Available from: https://diabetesatlas.org/en/.

[66] Lonardo A, Nascimbeni F, Mantovani A, Targher G. Hypertension, diabetes, atherosclerosis and NASH: Cause or consequence? J Hepatol 2018;68:335-352. doi:10.1016/j.jhep.2017.09.021.

[67] Muntner P, Carey RM, Gidding S, Jones DW, Taler SJ, Wright JT Jr, et al. Potential US population impact of the 2017 ACC/AHA high blood pressure guideline. Circulation 2018;137:109-118. doi:10.1161/CIRCULATIONAHA.117.032582

[68] Schiffrin EL. Global impact of the 2017 American College of Cardiology/ American Heart Association Hypertension Guidelines: A perspective From American Heart Association Hypertension Guidelines: A perspective From HA.117.032849.

[69] Wander GS, Ram CVS. Global impact of 2017 American Heart Association/ American College of Cardiology Hypertension Guidelines: A perspective from India. Circulation 2018;137:549-550. doi:10.1161/CIRCULATIONAHA.117.032877

[70] Wang JG, Liu L. Global impact of 2017 American College of Cardiology/ American Heart Association Hypertension Guidelines: A perspective from China. Circulation 2018;137:546-548. doi:10.1161/CIRCULATIONAHA.117.032890.

[71] Kim HC, Jeon YW, Heo ST. Global impact of the 2017 American College of Cardiology/American Heart Association Hypertension Guidelines. Circulation 2018;138:2312-2314. doi:10.1161/CIRCULATIONAHA.118.036312.

[72] Méndez-Sánchez N, Cerda-Reyes E, Higuera-de-la-Tijera F, Salas-García AK, Cabrera-Palma S, Cabrera-Álvarez G, et al. Dyslipidemia as a risk factor for liver fibrosis progression in a multicentric population with nonalcoholic steatohepatitis. F1000Res 2020;9:56. doi:10.12688/f1000research.21918.1.

[73] Raygor V, Abbasi F, Lazzeroni LC, Kim S, Ingelsson E, Reaven GM, et al. Impact of race/ethnicity on insulin resistance and hypertriglyceridaemia. Impact of race/ethnicity on insulin resistance and hypertriglyceridaemia.

[74] Frank AT, Zhao B, Jose PO, Azar KM, Fortmann SP, Palaniappan LP. Racial/ ethnic differences in dyslipidemia patterns. Circulation 2014;129:570579. doi:10.1161/CIRCULATIONAHA.113.005757.

[75] Golabi P, Otgonsuren M, de Avila L, Sayiner M, Rafiq N, Younossi ZM. Components of metabolic syndrome increase the risk of mortality in nonalcoholic fatty liver disease (NAFLD). Medicine (Baltimore) 2018;97:e0214. doi:10.1097/MD.0000000000010214.

[76] Huang Y, Cohen JC, Hobbs HH. Expression and characterization of a PNPLA3 protein isoform (I148M) associated with nonalcoholic fatty liver disease. ] protein isoform (I148M) associated with nonalcoholic fatty liver dise
Biol Chem 2011;286:37085-37093. doi:10.1074/jbc.M111.290114.

[77] Sookoian S, Pirola C]. Genetic predisposition in nonalcoholic fatty liver disease. Clin Mol Hepatol 2017;23:1-12. doi:10.3350/cmh.2016.0109.

[78] Wei JL, Leung JC, Loong TC, Wong GL, Yeung DK, Chan RS, et al. Prevalence and severity of nonalcoholic fatty liver disease in non-obese patients: A population study using proton-magnetic resonance spectroscopy. Am J
Gastroenterol 2015;110:1306-1314. doi:10.1038/ajg.2015.235.

[79] Romeo S, Kozlitina J, Xing C, Pertsemlidis A, Cox D, Pennacchio LA, et al. Genetic variation in PNPLA3 confers susceptibility to nonalcoholic fatty liver disease. Nat Genet 2008;40:1461-1465. doi:10.1038/ng.257.

[80] Mancina RM, Dongiovanni P, Petta S, Pingitore P, Meroni M, Rametta R, et al. The MBOAT7-TMC4 variant rs641738 increases risk of nonalcoholic fatty liver disease in individuals of European descent. Gastroenterology 2016;150:1219-1230.e6. doi:10.1053/j.gastro.2016.01.032.

[81] Kozlitina J, Smagris E, Stender S, Nordestgaard BG, Zhou HH, TybjærgHansen A, et al. Exome-wide association study identifies a TM6SF2 variant that confers susceptibility to nonalcoholic fatty liver disease. Nat Genet 2014;46:352-356. doi:10.1038/ng.2901.

[82] Beer NL, Tribble ND, McCulloch LJ, Roos C, Johnson PR, Orho-Melander $\mathrm{M}$, et al. The P446L variant in GCKR associated with fasting plasma glucose and triglyceride levels exerts its effect through increased glucokinase activity in liver. Hum Mol Genet 2009;18:4081-4088. doi:10.1093/hmg/ ddp357.

[83] Kallwitz ER, Tayo BO, Kuniholm MH, Cai J, Daviglus M, Cooper RS, et al. American ancestry is a risk factor for suspected nonalcoholic fatty liver disease in Hispanic/Latino adults. Clin Gastroenterol Hepatol 2019;17:23012309. doi:10.1016/j.cgh.2019.02.007.

[84] Valenti L, Dongiovanni P, Fracanzani AL, Santorelli G, Fatta E, Bertelli C, et al. Increased susceptibility to nonalcoholic fatty liver disease in heterozygotes for the mutation responsible for hereditary hemochromatosis. Dig Liver Dis 2003;35:172-178. doi:10.1016/s1590-8658(03)00025-2.

[85] Musso G, Gambino R, De Michieli F, Cassader M, Rizzetto M, Durazzo M, et al. Dietary habits and their relations to insulin resistance and postprandial lipemia in nonalcoholic steatohepatitis. Hepatology 2003;37:909-916. doi: 10.1053/jhep.2003.50132.

[86] Ryu S, Chang Y, Jung HS, Yun KE, Kwon MJ, Choi Y, et al. Relationship of sitting time and physical activity with non-alcoholic fatty liver disease. J Hepatol 2015;63:1229-1237. doi:10.1016/j.jhep.2015.07.010.

[87] Koopman KE, Caan MW, Nederveen A], Pels A, Ackermans MT, Fliers E, et al. Hypercaloric diets with increased meal frequency, but not meal size, increase intrahepatic triglycerides: a randomized controlled trial. Hepatology 2014;60:545-553. doi:10.1002/hep.27149.

[88] Abdelmalek MF, Suzuki A, Guy C, Unalp-Arida A, Colvin R, Johnson RJ, et al. Increased fructose consumption is associated with fibrosis severity in patients with nonalcoholic fatty liver disease. Hepatology 2010;51:19611971. doi:10.1002/hep.23535

[89] Lê KA, Tappy L. Metabolic effects of fructose. Curr Opin Clin Nutr Metab Care 2006;9:469-475. doi:10.1097/01.mco.0000232910.61612.4d.

[90] Browning JD, Baker JA, Rogers T, Davis J, Satapati S, Burgess SC. Shortterm weight loss and hepatic triglyceride reduction: evidence of a metabolic advantage with dietary carbohydrate restriction. Am J Clin Nutr 2011;93:1048-1052. doi:10.3945/ajcn.110.007674.

[91] Shim P, Choi D, Park Y. Association of blood fatty acid composition and dietary pattern with the risk of non-alcoholic fatty liver disease in patients who underwent cholecystectomy. Ann Nutr Metab 2017;70:303-311. doi:10.1159/000475605

[92] Chang Y, Ryu S, Sung E, Woo HY, Cho SI, Yoo SH, et al. Weight gain within the normal weight range predicts ultrasonographically detected fatty liver in healthy Korean men. Gut 2009;58:1419-1425. doi:10.1136/ gut.2008.161885.

[93] Wong VW, Wong GL, Yeung DK, Lau TK, Chan CK, Chim AM, et al. Incidence of non-alcoholic fatty liver disease in Hong Kong: a population study with paired proton-magnetic resonance spectroscopy. J Hepatol 2015;62:182paired proton-magnetic resonance spectrc
189. doi:10.1016/j.jhep.2014.08.041.

[94] Lutsey PL, Steffen LM, Stevens J. Dietary intake and the development of the metabolic syndrome: the Atherosclerosis Risk in Communities study. Circulation 2008;117:754-761. doi:10.1161/CIRCULATIONAHA.107.716159.

[95] Soleimani D, Ranjbar G, Rezvani R, Goshayeshi L, Razmpour F, Nematy M. Dietary patterns in relation to hepatic fibrosis among patients with nonalcoholic fatty liver disease. Diabetes Metab Syndr Obes 2019;12:315-324. doi:10.2147/DMSO.S198744.

[96] Chung GE, Youn J, Kim YS, Lee JE, Yang SY, Lim JH, et al. Dietary patterns are associated with the prevalence of nonalcoholic fatty liver disease in Korean adults. Nutrition 2019;62:32-38. doi:10.1016/j.nut.2018.11.021.

[97] Bian H, Hakkarainen A, Lundbom N, Yki-Järvinen H. Effects of dietary interventions on liver volume in humans. Obesity (Silver Spring) 2014;22:989995. doi:10.1002/oby.20623.

[98] Kani AH, Alavian SM, Esmaillzadeh A, Adibi P, Azadbakht L. Effects of a novel therapeutic diet on liver enzymes and coagulating factors in patients with non-alcoholic fatty liver disease: A parallel randomized trial. Nutrition 2014;30:814-821. doi:10.1016/j.nut.2013.11.008.

[99] Kalafati IP, Borsa D, Dimitriou M, Revenas K, Kokkinos A, Dedoussis GV. Dietary patterns and non-alcoholic fatty liver disease in a Greek case-control study. Nutrition 2019;61:105-110. doi:10.1016/j.nut.2018.10.032.

[100] Zhu FS, Liu S, Chen XM, Huang ZG, Zhang DW. Effects of $n-3$ polyunsaturated fatty acids from seal oils on nonalcoholic fatty liver disease associated with hyperlipidemia. World J Gastroenterol 2008;14:6395-6400. doi:10.3748/wjg.14.6395.

[101] Parker HM, Johnson NA, Burdon CA, Cohn JS, O'Connor HT, George J. Omega- 3 supplementation and non-alcoholic fatty liver disease: a systematic review and meta-analysis. J Hepatol 2012;56:944-951. doi:10.1016/j. jhep.2011.08.018.

[102] Ma J, Hennein R, Liu C, Long MT, Hoffmann U, Jacques PF, et al. Improved diet quality associates with reduction in liver fat, particularly in individuals with high genetic risk scores for nonalcoholic fatty liver disease. Gastroenterology 2018;155:107-117. doi:10.1053/j.gastro.2018.03.038.

[103] Sofi F, Macchi C, Abbate R, Gensini GF, Casini A. Mediterranean diet 
and health status: an updated meta-analysis and a proposal for a literature-based adherence score. Public Health Nutr 2014;17:2769-2782. doi:10.1017/S1368980013003169.

[104] Cantero I, Abete I, Babio N, Arós F, Corella D, Estruch R, et al. Dietary Inflammatory Index and liver status in subjects with different adiposity levels within the PREDIMED trial. Clin Nutr 2018;37:1736-1743. doi:10.1016/j. clnu.2017.06.027.

[105] Papamiltiadous ES, Roberts SK, Nicoll AJ, Ryan MC, Itsiopoulos C, Salim $A$, et al. A randomised controlled trial of a Mediterranean Dietary Intervention for Adults with Non Alcoholic Fatty Liver Disease (MEDINA): study protion for Adults with Non Alcoholic Fatty Liver Disease (MEDINA): study pro-
tocol. BMC Gastroenterol 2016;16:14. doi:10.1186/s12876-016-0426-3.

[106] Arciero PJ, Gentile CL, Pressman R, Everett M, Ormsbee MJ, Martin J, et al. Moderate protein intake improves total and regional body composition and insulin sensitivity in overweight adults. Metabolism 2008;57:757-765. doi:10.1016/j.metabol.2008.01.015.

[107] Park SY, Noureddin M, Boushey C, Wilkens LR, Setiawan VW. Diet quality association with nonalcoholic fatty liver disease by cirrhosis status: The multiethnic cohort. Curr Dev Nutr 2020;4:nzaa024. doi:10.1093/cdn/ nzaa024.

[108] Noureddin M, Zelber-Sagi S, Wilkens LR, Porcel J, Boushey C], Le Marchand $L$, et al. Diet associations with nonalcoholic fatty liver disease in an ethnically diverse population: The multiethnic cohort. Hepatology 2020;71:1940-1952. doi:10.1002/hep.30967.

[109] Zelber-Sagi S, Ivancovsky-Wajcman D, Fliss Isakov N, Webb M, Orenstein $\mathrm{D}$, Shibolet $\mathrm{O}$, et al. High red and processed meat consumption is associated with non-alcoholic fatty liver disease and insulin resistance. J Hepatol 2018;68:1239-1246. doi:10.1016/j.jhep.2018.01.015.

[110] Moore MP, Cunningham RP, Dashek RJ, Mucinski JM, Rector RS. A fad too far? Dietary strategies for the prevention and treatment of NAFLD. Obesity (Silver Spring) 2020;28:1843-1852. doi:10.1002/oby.22964.

[111] Johari MI, Yusoff K, Haron J, Nadarajan C, Ibrahim KN, Wong MS, et al. A randomised controlled trial on the effectiveness and adherence of modified alternate-day calorie restriction in improving activity of non-alcoholic fatty liver disease. Sci Rep 2019;9:11232. doi:10.1038/s41598-019-47763-8.
[112] Thoma C, Day CP, Trenell MI. Lifestyle interventions for the treatment of non-alcoholic fatty liver disease in adults: a systematic review. J Hepatol 2012;56:255-266. doi:10.1016/j.jhep.2011.06.010.

[113] Johnson NA, Sachinwalla T, Walton DW, Smith K, Armstrong A, Thompson MW, et al. Aerobic exercise training reduces hepatic and visceral lipids in obese individuals without weight loss. Hepatology 2009;50:1105-1112. doi:10.1002/hep.23129.

[114] Hallsworth K, Fattakhova G, Hollingsworth KG, Thoma C, Moore S, Taylor $R$, et al. Resistance exercise reduces liver fat and its mediators in non-alcoholic fatty liver disease independent of weight loss. Gut 2011;60:12781283. doi:10.1136/gut.2011.242073.

[115] Slentz CA, Bateman LA, Willis LH, Shields AT, Tanner CJ, Piner LW, et al. Effects of aerobic vs. resistance training on visceral and liver fat stores, liver enzymes, and insulin resistance by HOMA in overweight adults from STRRIDE AT/RT. Am J Physiol Endocrinol Metab 2011;301:E1033-E1039. doi:10.1152/ajpendo.00291.2011.

[116] Kim D, Kim WR. Nonobese fatty liver disease. Clin Gastroenterol Hepatol 2017;15:474-485. doi:10.1016/j.cgh.2016.08.028.

[117] Jin Y], Kim KM, Hwang S, Lee SG, Ha TY, Song GW, et al. Exercise and diet modification in non-obese non-alcoholic fatty liver disease: analysis of biopsies of living liver donors. J Gastroenterol Hepatol 2012;27:13411347. doi:10.1111/j.1440-1746.2012.07165.x.

[118] Hagström H, Nasr P, Ekstedt M, Hammar U, Stål P, Hultcrantz R, et al. Risk for development of severe liver disease in lean patients with nonalcoholic fatty liver disease: A long-term follow-up study. Hepatol Commun 2017:2:48-57. doi:10.1002/hep4.1124.

[119] Romero-Gómez M, Zelber-Sagi S, Trenell M. Treatment of NAFLD with diet, physical activity and exercise. J Hepatol 2017;67:829-846. doi:10.1016/j.jhep.2017.05.016.

[120] Setiawan VW, Wilkens LR, Lu SC, Hernandez BY, Le Marchand L, Henderson BE. Association of coffee intake with reduced incidence of liver cancer and death from chronic liver disease in the US multiethnic cohort. Gastroenterology 2015;148:118-125; quiz e15. doi:10.1053/j.gastro.2014.10.005 